\title{
Trapped surfaces in spherical expanding open universes.
}

\author{
Uwe Brauer*, Edward Malec ${ }^{\#+}$ and Niall Ó Murchadha*+ \\ *Max-Planck-Institut für Astrophysik, \\ Karl-Schwarzschild-Str. 1, \\ D-8046 Garching b. München, FRG \\ \# Institute of Physics, Jagellonian University \\ 30-064 Kraków, Reymonta 4, Poland \\ + Physics Department, University College \\ Cork, Ireland. \\ $5 / 11 / 1993$ \\ PACS numbers: 04.20., 98.80., 95.30., 97.60.
}

\begin{abstract}
Consider spherically symmetric initial data for a cosmology which, in the large, approximates an open $k=-1, \Lambda=0$ Friedmann-Lemaitre universe. Further assume that the data is chosen so that the trace of the extrinsic curvature is a constant and that the matter field is at rest at this instant of time. One expects that no trapped surfaces appear in the data if no significant clump of excess matter is to be found. This letter confirms this belief by displaying a necessary condition for the existence of trapped surfaces. This necessary condition, simply stated, says that a relatively large amount of excess matter must be concentrated in a small volume for trapped surfaces to appear.
\end{abstract}


Over the last few years, we have been investigating spherically symmetric solutions to the Einstein equations, both asymptotically flat [1] [2] [3] and cosmological [4], [5], with the intention of relating the amount of matter inside some chosen sphere to the possibility of trapped surfaces forming. The only situation which has not yet been dealt with is the case where we have a cosmological model with unbounded spatial slices which, on average, has negative scalar curvature. These are generalizations of the "open" (hyperboloidal) Friedmann universes. In this article we will derive a necessary condition for the appearance of a trapped surface in such a cosmology. This is very similar to equivalent necessary conditions we have derived in all the other cases. Interestingly enough, we have failed, in this open case, to find the matching sufficient condition which we were able to derive in all the other cases. We comment further on this at the end of this article.

Initial data for solutions to the Einstein equations consist of four objects (see [6]) $\left[\mathrm{rack} g_{a b}, K_{a b}, \rho\right.$ (energy density) and $J_{b}$ (matter current density)]rack; where $g_{a b}$ is a three dimensional, Riemannian (positive definite) metric which describes a three dimensional manifold $\Sigma ; \Sigma$ is to be regarded as a spacelike slice through the four manifold which is the solution of the Einstein equations and $K_{a b}$ is a symmetric three tensor which is the extrinsic curvature of $\Sigma$ as an embedded surface in the four-manifold. Consider a spacelike two surface $S$ embedded in $\Sigma$. If the surface is orientable, we can identify a unit vector $n^{a}$ as the "outgoing" direction orthogonal to $S$ in $\Sigma$. Now $J_{b} n^{b}$ measures the outflux of matter through $S$.

These data are not independent. They must satisfy the constraints,

$$
\begin{aligned}
{ }^{(3)} R[g]-K_{a b} K^{a b}+\left(K^{a}{ }_{a}\right)^{2}=16 \pi \rho & \text { (Hamiltonian constraint) } \\
D_{a} K^{a}{ }_{b}-D_{b} K^{a}{ }_{a}=-8 \pi J_{b} & \text { (momentum constraint) }
\end{aligned}
$$

where ${ }^{(3)} R[g]$ is the scalar curvature of $\Sigma, D$ the covariant derivative compatible with $g_{a b}$. The trace $\left(g^{a b} K_{a b}\right)$ of the second fundamental form $K_{a b}$ is equal to the (positive) rate of change of the 3 -dim volume in a timelike direction $\frac{d}{d t}(d V)=g^{a b} K_{a b} d V$.

Here we wish to consider initial data for a spherically symmetric cosmology which in the large looks like an open Friedmann universe. The standard time slice through an open (hyperboloidal) Friedmann-Lemaître universe is given 
by a hyperboloid of constant negative curvature where the line-element is given by

$$
d \widehat{s}^{2}=a^{2}(t)\left(d r^{2}+\sinh ^{2} r\left[d \theta^{2}+\sin ^{2} \theta d \varphi^{2}\right]\right)
$$

where $a$ is constant on $\Sigma$; $\widehat{K}_{a b}$ is pure trace $\widehat{K}_{a b}=H \widehat{g}_{a b}, H=\frac{d a}{d t} / \mathrm{a}$ is the Hubble constant; the (background) energy-density $\widehat{\rho}$ is a constant, $\widehat{J}^{a}=0$. We will use this data as a background against which we will compare our actual spherical solution. Background quantities will be denoted by a hat.

The Hamiltonian constraint (1) now gives

$$
-\frac{6}{a^{2}}+6 H^{2}=16 \pi \widehat{\rho}
$$

while the momentum constraint (2) is identically satisfied.

We wish to consider a spherically symmetric spacetime whose spatial topology agrees with that of the open Robertson-Walker geometry円. We focus on a spacelike slice through the spacetime which is itself spherically symmetric and on which the trace of the extrinsic curvature is a constant. This is not as restrictive a condition as it appears. If one is given a spacetime with a spatial symmetry, the $\operatorname{tr} K=$ const. slices respect this symmetry [7].

Since the three-metric is spherically symmetric we know that it is conformally flat and the line element can be written as

$$
d s^{2}=\phi^{4}(r) d \widehat{s}^{2}
$$

where $\phi \geq 0$. We can write the extrinsic curvature (assuming only spherical symmetry) as

$$
K_{a b}=H g_{a b}+\left(n_{a} n_{b}-\frac{g_{a b}}{3}\right) K(r)
$$

where $n^{a}$ is the unit radial vector. We further assume $J^{a}=J(r) n^{a}$.

Since $H$ is assumed constant, only $K(r)$ appears in the momentum constraint. As in the previous articles, the momentum constraint can be written as an explicit integral to give

$$
K\left(R_{S}\right)=\frac{-12 a \pi}{\phi^{6}\left(R_{S}\right) \sinh ^{3}\left(R_{S}\right)} \int_{0}^{R_{S}} \phi^{8}(r) \sinh ^{3}(r) J(r) d r .
$$

\footnotetext{
${ }^{1}$ If we refer to the geometrical (or kinematical) properties of this spacetime we use the term Robertson-Walker, while for dynamical aspects we use the term Friedmann-Lemaître.
} 
One immediate consequence of $(\overline{7})$ is that if the matter is at rest in the slice $(J=0)$ we have that $K=0$ and so the extrinsic curvature is pure trace. For the rest of this article we will assume that this holds. Therefore the momentum constraint is automatically satisfied and we need not consider it further.

To evaluate the Hamiltonian constraint we first need to calculate the scalar curvature of the metric. To do so we use the background metric $\widehat{g}_{a b}$ and that $\widehat{R}=-\frac{6}{a^{2}}$. We can use the fact that $g_{a b}=\phi^{4} \widehat{g}_{a b}$ to write

$$
{ }^{(3)} R[g]=-8 \phi^{-5} \widehat{\Delta} \phi-\frac{6}{a^{2}} \phi^{-4} .
$$

Hence the Hamiltonian constraint reads

$$
\phi^{-5} \widehat{\Delta} \phi=-2 \pi \delta \rho-\frac{3}{4 a^{2}}\left(1-\phi^{4}\right) \phi^{-4}
$$

where $\delta \rho$ denotes $\rho-\widehat{\rho}$. Let us stress here that we make no assumption that $\delta \rho$ is either small or has a fixed sign.

We wish to consider whether such a system will gravitationally collapse to form a black hole. Evidence would be the appearance of trapped surfaces in the initial data. Consider a spherically symmetric two surface $S$ in the initial data set. The expansion of outgoing null rays from this surface $S$ is given by

$$
\theta=D_{a} n^{a}+\left(g^{a b}-n^{a} n^{b}\right) K_{a b}
$$

where $n^{a}$ is the unit (spacelike) normal to this surface. If $\theta<0$ we say that the surface is (outer) trapped. One of the singularity theorems of general relativity [8], [9] tells us that the appearance of trapped surfaces is an indication that gravitational collapse is occuring. The expansion of a spherical surface in the open Friedmann universe is given by

$$
\theta=\widehat{D}_{a} \widehat{n}^{a}+2 H=\frac{2 \operatorname{coth} r+2 a H}{a} ; \quad \widehat{n}^{a}=\left(a^{-1}, 0,0\right) .
$$

Therefore if the universe is expanding $(H>0)$ no trapped surfaces exist.

In the spherically symmetric but non homogeneous model we are considering here it is easy to show that the expansion can be written as

$$
\theta=D_{a} n^{a}+2 H=\phi^{-3}\left(4 \phi^{\prime}+2 \phi \operatorname{coth} r\right)+2 H ; \quad n^{a}=\left(\phi^{-1} a^{-1}, 0,0\right)
$$


where $\phi^{\prime}=\frac{d \phi}{d r}$.

To relate this expression to the matter content of the universe one integrates (9) in the physical space over a sphere $S$ of coordinate radius $R_{S}$. Using spherical symmetry, integration by parts and Gauss law the left hand side gives

$$
\begin{aligned}
\int_{V} \phi^{-5} \widehat{\Delta} \phi d V & =\int_{V} \phi^{-5} \widehat{\Delta} \phi \phi^{6} d \widehat{V} \\
& =\left.4 \pi \phi \phi^{\prime} \sinh ^{2} r\right|_{R}-4 \pi \int_{0}^{R_{S}} \phi^{\prime 2} \sinh ^{2} r a d r .
\end{aligned}
$$

To do this integration one needs to use that $d V=\phi^{6} d \widehat{V}=\phi^{6} a^{3} \sinh ^{2} r \sin \varphi$. The right hand side of (9) gives

$$
\int_{V} \phi^{-5} \widehat{\Delta} \phi d V=2 \pi \delta M_{S}+\frac{3}{4 a^{2}} V-\frac{3}{4 a^{2}} 4 \pi \int_{0}^{R_{S}} \phi^{2} \sinh ^{2} r a d r .
$$

We equate (14) with (13), divide by $2 \pi$, recognize that the area $A$ of a sphere $S$ at a given radius $R$ is given by $A=\int_{S} \sqrt{|g|} d^{2} x=4 \pi a^{2} \phi^{4} \sinh ^{2} R_{S}$ and use (12) to finally get

$$
\begin{aligned}
\frac{A}{8 \pi} \theta= & -\delta M_{S}+\int_{0}^{R_{S}}\left[2 \phi^{\prime} \sinh ^{2} r+\left(\phi^{2} \cosh r \sinh r\right)^{\prime}-\frac{3}{2} \phi^{2} \sinh ^{2} r\right] a d r \\
& +\frac{3}{8 \pi a^{2}} V+\frac{H A}{4 \pi} \\
= & -\delta M_{S}+\int_{0}^{R_{S}}\left[\frac{1}{2} \phi^{2}+\frac{1}{2}\left(\phi \cosh r+2 \phi^{\prime} \sinh r\right)^{2}\right] a d r \\
& +\frac{3}{8 \pi a^{2}} V+\frac{H A}{4 \pi} .
\end{aligned}
$$

(15) is the appropriate equation to show a necessary condition; We assume $S$ to be trapped, i.e., $\theta<0$ which leads to

$$
0>-\delta M_{S}+\int_{0}^{R_{S}}\left[\frac{1}{2} \phi^{2}+\frac{1}{2}\left(\phi \cosh r+2 \phi^{\prime} \sinh r\right)^{2}\right] a d r+\frac{3}{8 \pi a^{2}} V+\frac{H A}{4 \pi}
$$




$$
\geq-\delta M_{S}+\frac{1}{2} L_{S}+\frac{3}{4 a^{2}} V+\frac{H A}{4 \pi} .
$$

The proper radius of the sphere of coordinate radius $R_{S}$ is given by

$$
L_{S}=\int_{0}^{R_{S}} \sqrt{g_{r r}} d r=\int_{0}^{R_{S}} \phi^{2} a d r
$$

Hence, if a given surface is trapped, we get

$$
\delta M_{S} \geq \frac{1}{2} L_{S}+\frac{3}{8 \pi a^{2}} V+\frac{H A}{4 \pi}
$$

Finally we have:

\section{Theorem 1 (A necessary condition)}

Assume one is given initial data for a spherically symmetric open cosmology, such that

$$
\begin{aligned}
& \text { 1.) } K_{a}^{a}=\text { const }=3 H \text {; } \\
& \text { 2.) } J_{b}=0 \text {, }
\end{aligned}
$$

and (background) Friedmann-Lemaître data (a, H, $\widehat{\rho}$ ) satisfying eqn.(4).

If for a spherical surface $S$, its proper radius $L_{S}$, its area $A$, the amount of the excess mass $\delta M_{S}$ and the volume $V$ inside $S$ satisfy

$$
\delta M_{S}<\frac{1}{2} L_{S}+\frac{A H}{4 \pi}+\frac{3 V}{8 \pi a^{2}}
$$

then $S$ is not trapped.

An obvious reorganization of Eqn.(19) can be achieved by moving the term $\hat{\rho} V$ from the left to the right hand side. We then can use Eqn.(4) to give

$$
\widehat{\rho} V+\frac{3}{8 \pi a^{2}} V=\frac{3 H^{2} V}{8 \pi}
$$

This gives us a condition for the nonappearance of trapped surfaces which is independent of any choice of background. 


\section{Theorem 2}

Assume one is given initial data as in Theorem 1.

If for a spherical surface $S$, its proper radius $L_{S}$, its area $A$, the total amount of matter $M_{S}$ enclosed by $S$ and the volume $V$ inside $S$ satisfy

$$
M_{S}<\frac{1}{2} L_{S}+\frac{A H}{4 \pi}+\frac{3 H^{2} V}{8 \pi}
$$

then $S$ is not trapped.

This result forms the basis of a simple test which may allow a determination of the global topology of our universe. [10

In the other cosmological models and in the case of asymptotically flat data we have found essentially identical necessary conditions and we have also found sufficient conditions expressed in terms of the same parameters. Surprisingly, we have failed to date to find, in the open case, a sufficient condition. This should not lead one to jump to the hasty conclusion that trapped surfaces are absent from spherical expanding open universes. It is easy to find examples with trapped surfaces. Because of the spherical symmetry, it is a simple matter to construct a model which consists of a constant density sphere embedded in an open cosmology. If the density is large enough, trapped surfaces appear.

\section{References}

[1] P. Bizon, E. Malec, and N. Ó Murchadha. Trapped Surfaces in Spherical Stars. Physical Review Letters, 61(10):1147-1150, September 1988.

[2] P. Bizon, E. Malec, and N. Ó Murchadha. Trapped surfaces due to concentration of matter in spherically symmetric geometries. Classical and Quantum Gravity, 6:961-976, May 1989.

[3] P. Bizon, E. Malec, and N. Ó Murchadha. Binding energy for spherical stars . Classical and Quantum Gravity, 7:1953-1959, May 1990.

[4] Uwe Brauer and Edward Malec. Trapped surfaces in expanding open universes . Physical Review D, 45(6):R1836-39, March 1992. 
[5] E. Malec and N. Ó Murchadha. Trapped Surfaces and spherical closed cosmologies . Physical Review D, 47(2):1454-1464, February 1993.

[6] C. W. Misner, K. Thorne, and J. Wheeler. Gravitation. Freeman, San Francisco, 1973.

[7] N. Ó Murchadha. to be published, 1993.

[8] Roger Penrose. Techniques of Differential Topology in Relativity. Society for Industrial and Applied Mathematics, Philadelphia, 1972.

[9] Steven W. Hawking and G.R.W. Ellis. The large scale structure of spacetime . Cambridge University Press, Cambridge, 1973.

[10] E. Malec and N. Ó Murchadha. Is the universe open or closed? Acta Cosmologica, XIX, 93 - 101, 1993. 